Archive for

Organic Chemistry

Arkivoc 2019, part v, 19-29

\title{
Ring closing metathesis and metal-catalyzed cyclopropanation for the preparation of sultone derivatives
}

\author{
Korany A. Ali ${ }^{* a, b}$ and Peter Metz \\ ${ }^{a}$ Applied Organic Chemistry Department, National Research Centre, 12622 Dokki, Giza, Egypt \\ ${ }^{b}$ Center of Excellence and advanced material- Nanotechnology Group, National Research Centre, 12622 Dokki, \\ Giza, Egypt \\ 'Organische Chemie I, Technische Universität Dresden, Bergstrasse 66, 01069 Dresden, Germany \\ Email: kornykhlil@gmail.com
}

Received 09-12-2018

Accepted 11-15-2018

Published on line $12-30-2018$

\section{Abstract}

Ring closing metathesis (RCM) using Grubbs catalyst $2^{\text {nd }}$ generation as a catalyst was applied to prepare series of novel unsaturated sultones with high yields. Many attempts, were applied for the cyclopropanation of the allylic sultones by Simmon-smith cyclopropanation using diethyl zinc/diiodomethane or $\mathrm{Zn}-\mathrm{Cu} /$ diiodomethane but in each case the corresponding cyclic adduct was not formed. A novel palladium or preferably rhodiumcatalyzed cyclopropanation of unsaturated sultones with ethyl diazoacetate was achieved by the transition metal-catalyzed transfer of a $\mathrm{CH}-\mathrm{CO}_{2} \mathrm{Et}$ unit. The reaction was applied by a portion-wise addition of ethyl diazoacetate over $6 \mathrm{~h}$ to a mixture of the sultones and palladium(II) acetate or rhodium(II) acetate dimer under low temperature $\left(0-20^{\circ} \mathrm{C}\right)$. The desired products of the cyclopropanation were achieved in each case, as a single diastereomer with $33-37 \%$ yield in the allylic sultones and $10 \%$ for vinylic sultone.

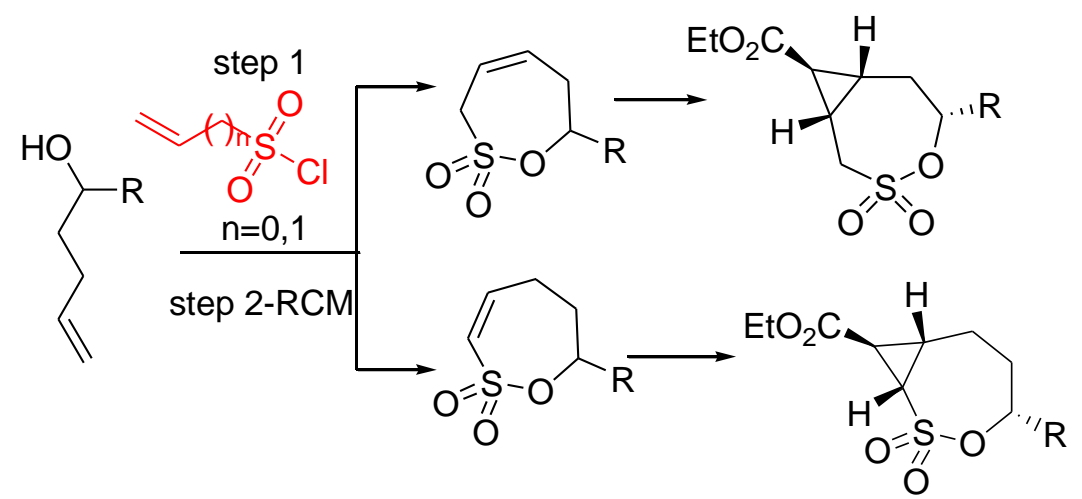

Keywords: Ring closing metathesis (RCM), carbenes generation; sultones, cyclopropanation 


\section{Introduction}

Sultones are important scaffolds in organic and bioorganic chemistry with good contribution in the synthesis of different types of heterocycles. ${ }^{1-6}$ Additionally, Sultones act as sulfoalkylating agents in organic and natural product synthesis ${ }^{7,8}$. Additionally, several publications have reported several biological activities of the sultones that includes antitumor and antiviral (anti-HIV) activities. ${ }^{9-12}$

On the other hand, a great deal of interest has been focused on the cyclopropanes as they have a wide contribution in the natural products and the biologically active synthetic compounds. ${ }^{13-16}$ Furthermore, the ring strain of the o-bond in the cyclopropane ring make cyclopropane derivatives highly reactive in transition metal catalyzed reactions.

The ring closing metathesis (RCM) and organometallic catalysis using Pd, Rh and Ru compounds are very important tools for organic chemistry and pharmaceutical applications as they used for the preparation of active ingredients in the field of organic chemistry and pharmaceutical industry. ${ }^{15-22}$

From these observations and in continuation of our interest of the chemistry of organo-sulfur heterocycles and sultones chemistry, ${ }^{22-26}$ we reported herein an interesting preparation of a series of unsaturated sultones in addition to successful attempts of metal-catalyzed cyclopropanation on the sultone scaffold.

\section{Results and Discussion}

In this study, several allylic and vinylic sultones were prepared from the appropriate sulfonate and Subsequent RCM. The unsaturated sulfonates (3) were prepared from the reaction of the primary or secondary alkenols $(\mathbf{2 a}, \mathbf{b})$ with allyl sulfonyl chloride (1), in the presence of TEA, under low temperature. We apply our modified procedure to obtain high yields of the sulfonates (3) via completing the addition process of the reactant under $-20^{\circ} \mathrm{C}$ and left the reaction under this temperature for $4 \mathrm{~h}$ (Scheme 1 ).

RCM was achieved by portion wise addition of Grubbs catalyst $2^{\text {nd }}$ generation under reflux temperature on a solution of the unsaturated sulfonate $\mathbf{3 a}$ or $\mathbf{3} \mathbf{b}$ to afford the corresponding sultone $(\mathbf{4} \mathbf{a}, \mathbf{b})$. the structures of the sultones $(\mathbf{4 a}, \mathbf{b})$ were confirmed by the elemental analysis and spectral data. The ${ }^{1} \mathrm{H}$-NMR spectrum of the $4 \mathrm{~b}$ revealed doublet signal at $1.35 \mathrm{ppm}$ corresponding to $\mathrm{CH}_{3}$, multiplets near $5.63,6.01 \mathrm{ppm}$ for 2 allylic $\mathrm{CH}$. Since there was only a single publication on the intermolecular cyclopropanation of vinylic ${ }^{27}$ or allylic sultones prior to our work we decided to investigate this transformation.

Many attempts, in this study, were applied for the cyclopropanation of the sultones $\mathbf{4 a , b}$ by SimmonsSmith cyclopropanation using diethyl zinc/diiodomethane or $\mathrm{Zn}-\mathrm{Cu} /$ diiodomethane, but in each case the cyclopropanation for the corresponding cyclic adduct was not obtained (Scheme 1).

The successful attempts and the first investigation for the cyclopropanation of the sultones $\mathbf{4 a , b}$ with ethyl diazoacetate to give the desired products as single diastereomers were achieved by palladium(II) acetate or rhodium(II) acetate dimer as a catalyst. The cyclopropanation reaction was achieved via transition metalcatalyzed transfer of a $\mathrm{CH}-\mathrm{CO}_{2} \mathrm{Et}$ unit under low temperature $0-20{ }^{\circ} \mathrm{C}$. with continuous and slow addition of ethyl diazoacetate over $6 \mathrm{~h}$.

The yields of the cyclopropanation adducts $\mathbf{6 a , b}$ were 33 and $37 \%$ in the case of rhodium (II) acetate dimer that was higher than the using of palladium(II) acetate with yield 15 and $17 \%$, respectively (Scheme 1 ). The structure of the bicyclic compounds $\mathbf{6 a}, \mathbf{b}$ were confirmed by the elemental analysis and spectral data. The relative configuration of $6 \mathbf{a}, \mathbf{b}$ was assigned by $2 \mathrm{D}$ NOESY analysis. The ${ }^{1} \mathrm{H}-\mathrm{NMR}$ spectrum of the $6 \mathbf{6}$ revealed 
signals at $1.25\left(\mathrm{CH}_{3}\right), 1.52-1.62(2 \mathrm{CH}), 1.90(\mathrm{CH}), 2.10-2.33(2 \mathrm{CH}), 2,33(\mathrm{CH}), 3.01(\mathrm{CH}), 3.81(\mathrm{CH}), 4.10\left(\mathrm{CH}_{2}\right)$, and $4.95(\mathrm{CH})$ ppm.

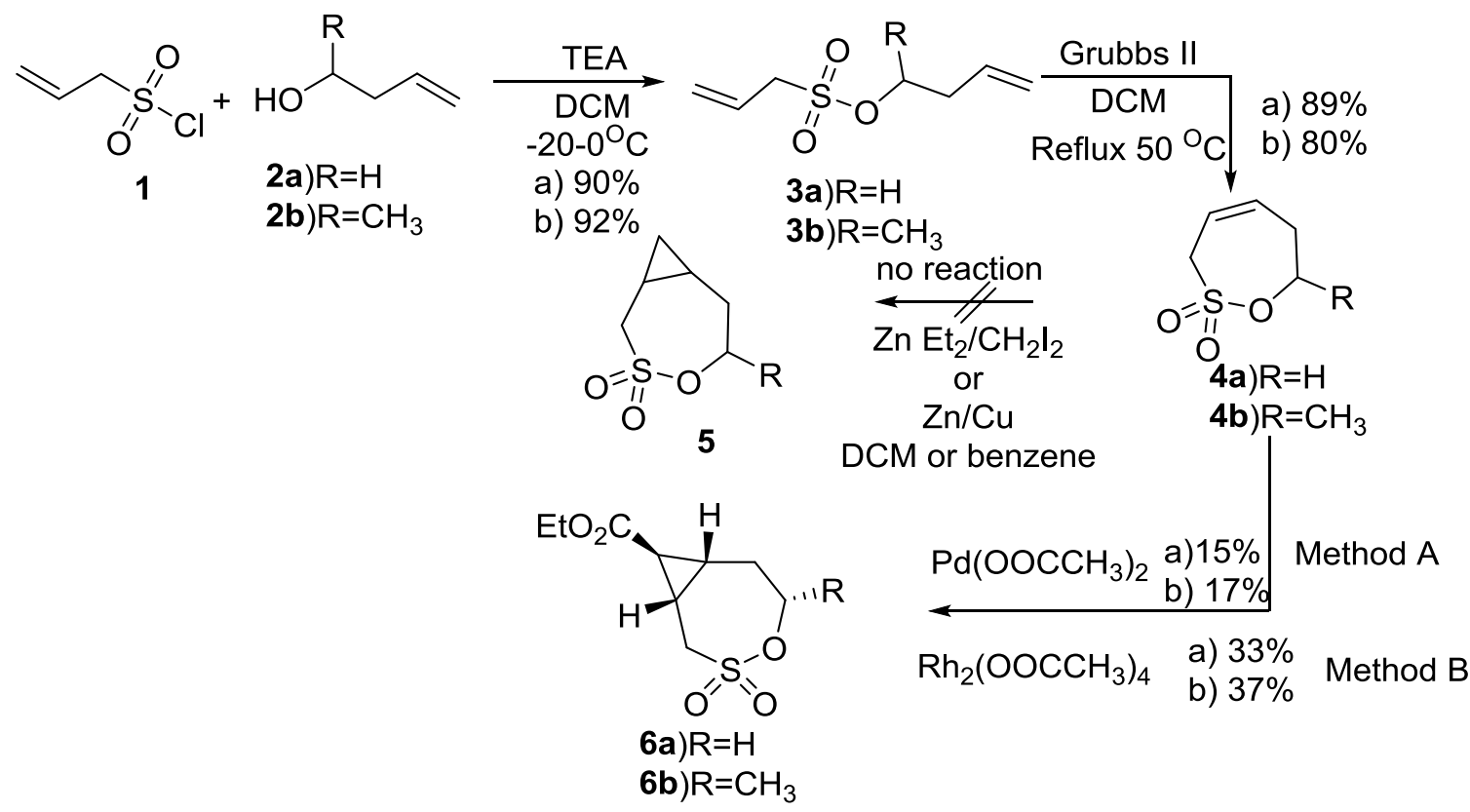

\section{Scheme 1}

To complete the overall picture for the preparation of novel sultones we have designed the synthetic pathway to prepare new vinyl sultones as presented in scheme 2. Firstly, the secondary allylic alcohols were prepared from the corresponding ketone using $\mathrm{LiAlH}_{4}$. Ethenesulfonyl chloride (10) was prepared in-situ from the treatment of chloro ethyl sulfonyl chloride (9) with TEA under low temperature. The unsaturated sulfonate derivatives (11) were readily prepared by esterification of the secondary alcohols (8) with the in-situ generated ethenesulfonyl chloride (10) in the presence of TEA under low temperature $\left(-40^{\circ} \mathrm{C}\right)$. The structures of the formed sulfonates were confirmed by elemental analysis and spectral data.

In this study, the second new series of sultones is the vinyl sultones (12) prepared by treatment of the freshly prepared vinyl sulfonate (11) with Grubbs(II) catalyst that added portion wise under reflux temperature (Scheme 2). The structures of the new vinyl sultones were confirmed by elemental analysis and spectral data. (See the experimental part).

Several attempts were applied for the cyclopropanation of the vinyl sultones 12 using sulfur yields; trimethylsulfonium bromide $\left(\mathrm{CH}_{3}\right)_{3} \mathrm{~S}^{+} \mathrm{Br}^{-}$, in the presence of potassium tert-butoxide in DMF but in each case lead to decomposition of the sultones due to the base; potassium tert-butoxide.

Cyclopropanation of the vinyl sultones $\mathbf{1 2 b}$, was achieved with very low yield (10\%) by treatment with ethyl diazoacetate in the presence of dirhodium(II) tetra acetate as a catalyst under low temperature $0-20^{\circ} \mathrm{C}$. the cyclopropanation was not achieved by palladium(II) acetate as catalyst (Scheme 2 ).

The constitution of the bicyclic sultone $\mathbf{1 4}$ was confirmed by the elemental analysis and spectral data, while the relative configuration was tentatively assigned in analogy to sultones 6 . The ${ }^{1} \mathrm{H}-\mathrm{NMR}$ spectrum of the sultone 14 revealed presence of 3 characteristic bands multiplets near $0.97,1.15$ and 1.70 ppm corresponding to the cyclopropane protons; $6-\mathrm{H}, 4-\mathrm{H}$, and $5-\mathrm{H}$, respectivly. Two signals that are charactarestic for the ester group were appeared as triplet and quartet at near 1.10 and 4.22 ppm, respectivly. 


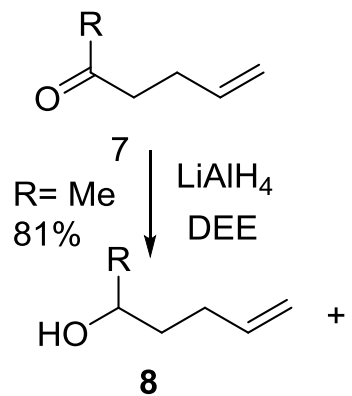

$\mathrm{R}=\mathrm{Me}, \mathrm{Et}$<smiles>O=S(=O)(Cl)CCCl</smiles><smiles>C=C(O)[As](=O)(=O)Cl</smiles>

10

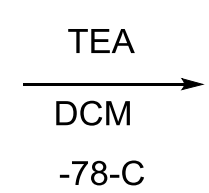

a) $79 \%$

b) $67 \%$<smiles>[R]C(CCC=C)OS(=O)(=O)C=C</smiles>

Grubbs II DCM Reflux $50^{\circ} \mathrm{C}$

$75 \%$

b) $71 \%$<smiles>[R]C1CCC2CC2S(=O)(=O)O1</smiles>

13
$\left(\mathrm{CH}_{3}\right)_{3} \mathrm{~S} \mathrm{Br}$<smiles>CC1=C(C)CCCC1</smiles><smiles>[R]C1CCC=CS(=O)(=O)O1</smiles>

14

\section{Scheme 2}

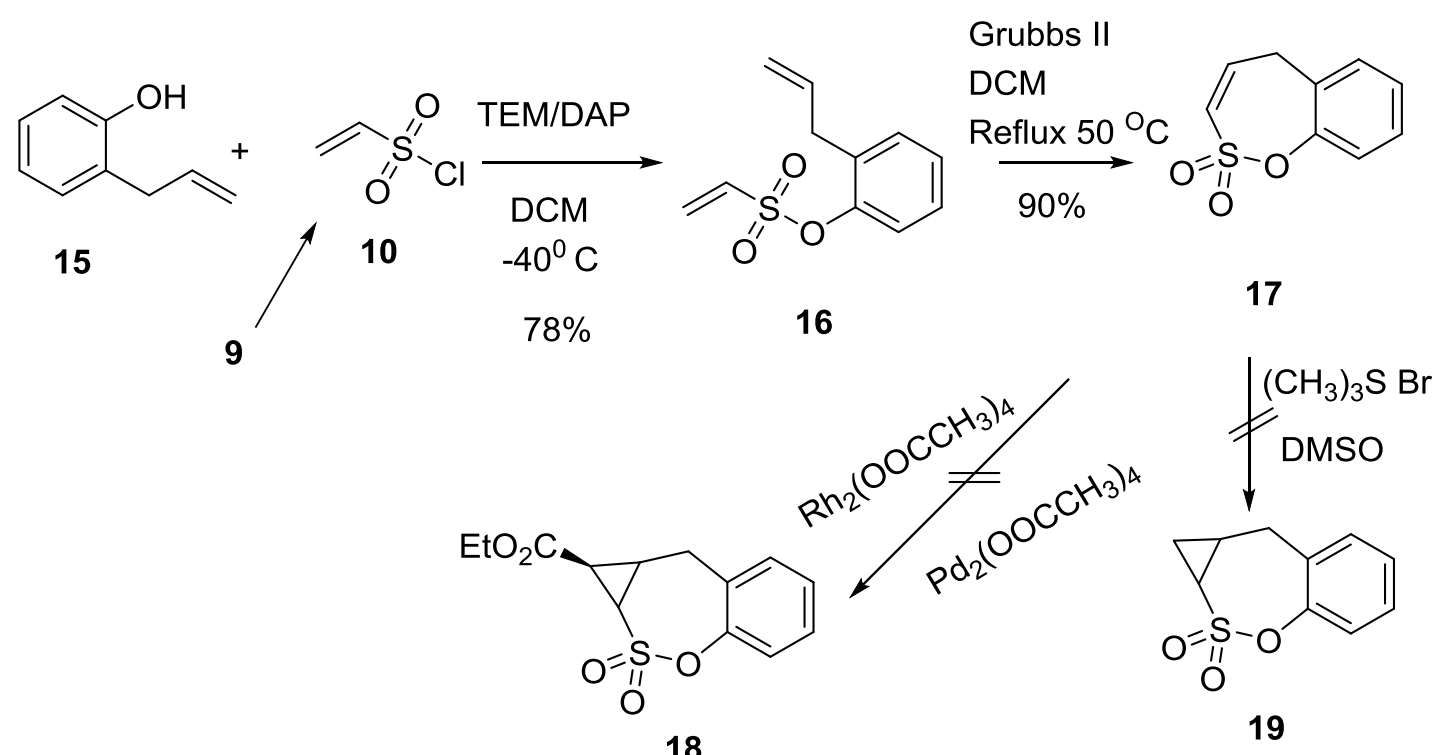

\section{Scheme 3}

New vinylic sultone constructed on benzene scaffold was obtained by RCM as presented in scheme 3 . The first step was the preparation of the unsaturated sulfonate $\mathbf{1 6}$ that was readily obtained by esterification of 
the 2-allylphenol (15) with ethenesulfonyl chloride (10), in the presence of TEA under low temperature (0-40). The unsaturated sulfonate derivative (16) was formed with $78 \%$ yield and its structure was confirmed by elemental analysis and spectral data. The vinyl sultone (17) was prepared with $90 \%$ yield by treatment the sulfonate 16 with Grubbs catalyst $2^{\text {nd }}$ generation under reflux temperature in DCM (Scheme 3 ).

The ${ }^{1} \mathrm{H}-\mathrm{NMR}$ spectrum of the sultone $(17)$ revealed bands at $3.72(\mathrm{~m}), 6.35(\mathrm{~m})$ and $6.48(\mathrm{~m}) \mathrm{ppm}$ corresponding to $\mathrm{CH}_{2}$ and $2 \mathrm{CH}$, respectivly. The aromatic protons apperd at 7.21-7.45 ppm.

The structure of the sultone (17) was fully confirmed by the X-ray single crystal analysis (Figure 1).

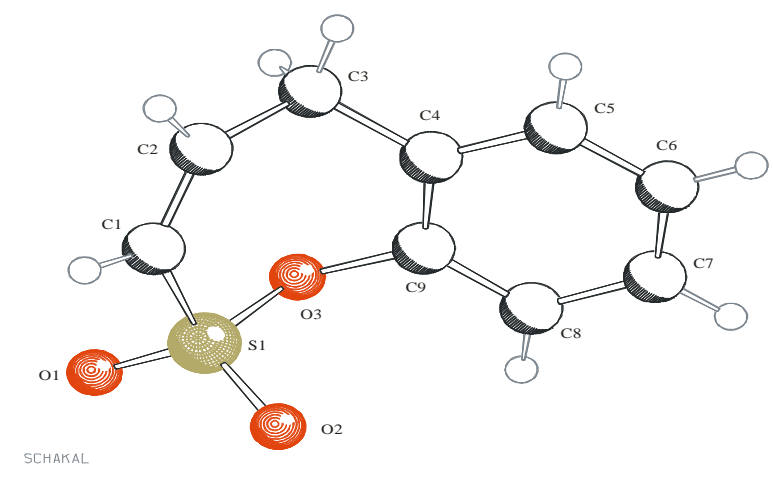

Figure 1: X-ray crystal structure of compound 17

We have tried to apply the cyclopropanation reaction on the later sultone by different treatments with ethyl diazoacetate in the presence of $\mathrm{Pd}(\mathrm{OAc})_{2}$ or $\mathrm{Rh}_{2}(\mathrm{OAc})_{4}$ but in each case there was no reaction.

\section{Conclusions}

In this study, a novel palladium- or preferably rhodium-catalyzed cyclopropanation of unsaturated sultones with ethyl diazoacetate to give the desired products as a single diastereomer have been achieved. These densely functionalized heterocycles are surely highly interesting intermediates for the preparation of pharmaceutically active compounds. The unsaturated sultones required for these investigations were readily available by esterification of unsaturated alcohols with sulfonyl chlorides and subsequent ruthenium-catalyzed ring-closing metathesis. With a range of novel sultones synthesized in this fashion, we first investigated different conditions for cyclopropanation using the Simmons-Smith methodology and also sulfur ylide chemistry to transfer an un-substituted methylene unit to the alkene moiety of the substrates. Whereas these reactions did not work with the unsaturated sultones at hand, the transition metal-catalyzed transfer of a $\mathrm{CH}$ $\mathrm{CO}_{2} \mathrm{Et}$ unit finally succeeded as detailed above. Probably, the cyclopropanation of the vinylic sultones was more difficult than the allylic sultones due to the high electron deficiency of the alkenes of the vinylic sultone. But in case of the allylic sultones where the separation of the sulfonyl group and the olefin by $\mathrm{CH}_{2}$ group might allow a [2+1] cycloaddition to give the desired cyclopropanes 


\section{Experimental Section}

General. Infrared spectra were recorded on a THERMONICOLET Avatar 360 instrument using ATR. NMR spectra were recorded on a Bruker AC $300 \mathrm{P}\left(300 \mathrm{MHz}{ }^{1} \mathrm{H}, 75 \mathrm{MHz}{ }^{13} \mathrm{C}\right)$, on a Bruker DRX $500 \mathrm{P}\left(500 \mathrm{MHz}{ }^{1} \mathrm{H}\right.$, $\left.125 \mathrm{MHz}{ }^{13} \mathrm{C}\right)$ or on a Bruker $\mathrm{AC} 600-\mathrm{P}\left(600 \mathrm{MHz}{ }^{1} \mathrm{H}, 151 \mathrm{MHz}{ }^{13} \mathrm{C}\right)$ spectrometer. Chemical shifts $(\delta)$ are quoted in parts per million ( $\mathrm{ppm}$ ) downfield of tetramethylsilane, using residual proton-containing solvent as internal standard $\left(\mathrm{CDCl}_{3}\right.$ at $\left.7.26 \mathrm{ppm}\right)$. Abbreviations used in the description of resonances are: $\mathrm{s}$ (singlet), $d$ (doublet), $\mathrm{t}$ (triplet), q (quartet), br (broad). Coupling constants $(J)$ are quoted to the nearest $0.1 \mathrm{~Hz}$. Mass spectra were recorded with an Agilent 5973N detector coupled with an Agilent 6890N GC (GC-MS, 70 eV) or else with a Bruker Esquire-LC (direct injection as a methanolic $\mathrm{NH}_{4} \mathrm{OAc}$ solution, ESI). HRMS spectra were recorded on a Bruker Daltonic "Impact II" (ESI-TOF). Elemental analysis was performed on a Hekatech EA 3000.

Preparation of prop-2-ene-1-sulfonyl chloride (1). Prop-2-ene-1-sulfonyl chloride (1) was prepared by our modification on the reported method $^{28}$ as in the following: To a stirred solution of allyl bromide $(12.10 \mathrm{~g}$, $0.1 \mathrm{~mol})$ in $100 \mathrm{~mL}$ distilled water was added $\mathrm{Na}_{2} \mathrm{SO}_{3}(15 \mathrm{~g}, 0.12 \mathrm{~mol})$. The reaction mixture was refluxed for $8 \mathrm{~h}$ then left to cool to room temperature. The reaction mixture was washed with $\mathrm{Et}_{2} \mathrm{O}$ several times and the aqueous layer was evaporated under reduced pressure. The crude product was washed with methanol, filtered off and left to dry to afford $(10.2 \mathrm{~g}, 0.07 \mathrm{~mol}, 71 \%$ yield) of sodium allysulfonate. The latter crude product was stirred at zero temperature with $30 \mathrm{~mL}$ of $\mathrm{POCl}_{3}$ for $1 / 2 \mathrm{~h}$ then refluxed for $5 \mathrm{~h}$ and left to cool to room temperature. $100 \mathrm{~mL}$ of THF was then added to the mixture then filtered off and washed several times with dry THF. The filtrate was evaporated carefully on rotavapor and the residue was distilled under vacuum ( 5 mbar). Allylsulfonyl chloride was separated with boiling point range $37-43^{\circ} \mathrm{C}$ as coreless oil (71\%).

General procedure for the preparation of but-3-enyl prop-2-ene-1-sulfonate derivatives (3a,b). To a stirred solution of the unsaturated alcohol (2a,b) (1.0 equiv.) and dry TEA (1.5 equiv.) in $\mathrm{CH}_{2} \mathrm{Cl}_{2}$ at low temperature ($20{ }^{\circ} \mathrm{C}$ ) was added ally sulfonyl chloride (1.2 equiv.) in $\mathrm{CH}_{2} \mathrm{Cl}_{2}$ drop by drop. The reaction mixture was left to stir under this temperature for $4 \mathrm{~h}$ then left to stir for $0.5 \mathrm{~h}$ at room temperature. Pentane was then added and the mixture was filtered through Celite. The filtrate was concentrated under reduced pressure and the crud residue was purified by flash chromatography.

General procedure for the preparation of hex-5-en-2-yl ethane sulfonate derivatives (11a,b) and 2allylphenyl ethenesulfonate (16). A solution of the unsaturated alcohol (7a,b) (1.0 equiv.) and dry TEA (1.5 equiv.) in $\mathrm{CH}_{2} \mathrm{Cl}_{2}$ was stirred at low temperature $\left(-40-0{ }^{\circ} \mathrm{C}\right)$ under inert atmosphere in two necked flask. $A$ solution of 2-chloroethanesulfonyl chloride (1.3-1.5 equiv.) in $\mathrm{CH}_{2} \mathrm{Cl}_{2}$ was then added drop by drop. The reaction mixture left to stir under this temperature for 3-7 $\mathrm{h}$. then diluted with pentane and filtered through Celite. The filtrate was concentrated under reduced pressure and the crud residue was purified by flash chromatography.

General procedure for the preparation of sultones $4 a, b, 12 a, b$ and 17 . To a stirred solution of the sulfonates; 3a, 3b, 12a, 12b or 16 (1eq) in $\mathrm{CH}_{2} \mathrm{Cl}_{2}$, in three necked flask under argon atmosphere, was added Grubbs catalyst $2^{\text {nd }}$ generation (0.03-0.05 equiv.) in three portions. The reaction mixture was refluxed with stirring for 5-12 h. the solvent was evaporated under reduced pressure and the solid crude product was purified by column chromatography (silica gel isohexane/ethyl acetate $9 / 1$ or diethyl ether/pentane 1/1) to afford the corresponding sultones $\mathbf{4 a}, \mathbf{4 b}, \mathbf{1 2 a}, \mathbf{1 2 b}$ and $\mathbf{1 7}$, respectively.

General procedure for the rhodium catalyzed cyclopropanation using ethyl diazoacetate. To a stirred solution of the sultone $\mathbf{4 a}, \mathbf{4 b}, \mathbf{1 2 a}, \mathbf{1 2} \mathbf{b}$ and $\mathbf{1 7}$, respectively (1eq) and the appropriate catalyst; palladium 
diacetate or rhodium(II) acetate dimer (1-3 mol\%) in $\mathrm{CH}_{2} \mathrm{Cl}_{2}$ under argon atmosphere, was added ethyl diazoacetate (2-3 equiv.) in DCM over $6 \mathrm{~h}$ period using syringe pump. The solvent was evaporated under reduced pressure and the solid crude product was purified by column chromatography (silica gel isohexane/ethyl acetate 9/1) to afford the corresponding 3-oxa-2-thiabicyclo[5.1.0]octane 2,2-dioxide.

But-3-enyl prop-2-ene-1-sulfonate (3a). Compound 3a was prepared according the general procedure from prop-2-ene-1-sulfonyl chloride (1) $(2.1 \mathrm{~g}, 15 \mathrm{mmol})$, but-3-en-1-ol (2a) (1g, $13.86 \mathrm{mmol})$ and TEA (2 mL). Colorless oil $\mathrm{R}_{f}: 0.53$ (Et ${ }_{2} \mathrm{O} /$ pentane; $\left.1 / 1\right)^{29}$; Yield (2.44g, 90\%). ${ }^{1} \mathrm{H} \mathrm{NMR}\left(\mathrm{CDCl}_{3}, 300 \mathrm{MHz}\right): \delta_{\mathrm{H}} 2.32-2.48(\mathrm{~m}, 2 \mathrm{H}$, $\left.\mathrm{CH}_{2}\right), 3.75\left(\mathrm{~d}, \mathrm{~J} 7.2 \mathrm{~Hz}, 2 \mathrm{H}, \mathrm{CH}_{2}\right), 4.33\left(\mathrm{t}, 2 \mathrm{H}, \mathrm{CH}_{2}\right), 5.05-5.15(\mathrm{~m}, 2 \mathrm{H}, 2 \mathrm{CH}), 5.40-5.51(\mathrm{~m}, 2 \mathrm{H}, 2 \mathrm{CH}), 5.71-5.95$ (m, 2H, 2CH).

Pent-4-en-2-yl prop-2-ene-1-sulfonate (3b). Compound $\mathbf{3 b}$ was prepared according the general procedure from prop-2-ene-1-sulfonyl chloride (1) (3 g, $21.4 \mathrm{mmol})$, pent-1-en-2-ol (2b) (1.5 g, $17.4 \mathrm{mmol})$ and TEA (3 $\mathrm{mL})$. Colorless oil $\mathrm{R}_{f}$ : 0.65 (Et ${ }_{2} \mathrm{O} /$ pentane; 1/1); Yield (3.03 g, 92\%). IR (KBr, vmax, cm $\left.{ }^{-1}\right): 3080,2982,2935$, $1643,1424,1336,1258,1162,992,904 .{ }^{1} \mathrm{H} \mathrm{NMR}\left(\mathrm{CDCl}_{3}, 300 \mathrm{MHz}\right): \delta(\mathrm{ppm})=1.34\left(\mathrm{~d}, J 6.2 \mathrm{~Hz}, 3 \mathrm{H}, \mathrm{CH}_{3}\right), 2.28-$ $2.47\left(\mathrm{~m}, 2 \mathrm{H}, \mathrm{CH}_{2}\right), 3.73\left(\mathrm{~d}, \mathrm{~J} 7.2 \mathrm{~Hz}, 2 \mathrm{H}, \mathrm{CH}_{2}\right), 4.72-4.83(\mathrm{~m}, 1 \mathrm{H}, \mathrm{CH}), 5.04-5.13\left(\mathrm{~m}, 2 \mathrm{H}, \mathrm{CH}_{2}\right), 5.33-5.43(\mathrm{~m}, 2$ $\left.\mathrm{H}, \mathrm{CH}_{2}\right), 5.63-5.91(\mathrm{~m}, 2 \mathrm{H}, 2 \mathrm{CH}) .{ }^{13} \mathrm{C} \mathrm{NMR}(\mathrm{CDCl} 3,75 \mathrm{MHz})(\delta \mathrm{ppm}): 20.66(\mathrm{q}), 40.95(\mathrm{t}), 55.76(\mathrm{t}), 79.31(\mathrm{~d})$,

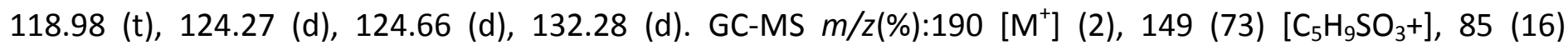
[ $\left.\mathrm{C}_{5} \mathrm{H}_{9} \mathrm{O}+\right], 69$ (100) [ $\left.\mathrm{C}_{5} \mathrm{H}_{9}+\right], 68$ (37), 67 (37), 53 (11), 45 (7) [ $\left.\mathrm{C}_{2} \mathrm{H}_{5} \mathrm{O}+\right]$. Anal. Calcd for $\mathrm{C}_{8} \mathrm{H}_{14} \mathrm{O}_{3} \mathrm{~S}$ (190.26): C, $50.50 ; \mathrm{H}, 7.42 ; \mathrm{S}, 16.85$. Found: $\mathrm{C}, 50.65 ; \mathrm{H}, 7.68 ; \mathrm{S}, 16.67$.

6,7-Dihydro-3H-1,2-oxathiepine 2,2-dioxide (4a). Compound $4 a$ was prepared according the general procedure from but-3-enyl prop-2-ene-1-sulfonate (3a) $(1.76 \mathrm{~g}, 10 \mathrm{mmol})$ and Grubbs catalyst 2nd generation $(0.26 \mathrm{~g}, 0.03 \mathrm{eq})$ as a colorless crystals (Et $2 \mathrm{O} /$ Pentane:1/1); Yield $(89 \%) .{ }^{28}{ }^{1} \mathrm{H} \mathrm{NMR}\left(\mathrm{CDCl}_{3}, 300 \mathrm{MHz}\right): \delta_{\mathrm{H}} 2.55-$ $2.60\left(\mathrm{~m}, 2 \mathrm{H}, \mathrm{CH}_{2}\right), 4.11-4.22\left(\mathrm{~m}, 2 \mathrm{H}, \mathrm{CH}_{2}\right), 4.51-4.60\left(\mathrm{~d}, 2 \mathrm{H}, \mathrm{CH}_{2}\right), 5.75-5.80(\mathrm{~m}, 1 \mathrm{H}, \mathrm{CH}), 6.23(\mathrm{~m}, 1 \mathrm{H}, \mathrm{CH})$.

7-Methyl-6,7-dihydro-3H-1,2-oxathiepine 2,2-dioxide (4b). Compound $\mathbf{4 b}$ was prepared according the general procedure from pent-4-en-2-yl prop-2-ene-1-sulfonate (3b) $(0.95 \mathrm{~g}, 5 \mathrm{mmol})$ and Grubbs catalyst 2nd generation $(0.13 \mathrm{~g}, 0.03 \mathrm{eq})$ as a colorless crystals; yield $(0.65 \mathrm{~g}, 80 \%)$; colorless crystals, $\mathrm{R}_{f}$ : 0.42 $\left(\mathrm{Et}_{2} \mathrm{O} /\right.$ Pentane:1/1). IR (KBr, vmax, $\left.\mathrm{cm}^{-1}\right): 2981,1453,1349,1326,1154,1038,898,755,671 .{ }^{1} \mathrm{H} \mathrm{NMR}(300$ $\left.\mathrm{MHz}, \mathrm{CDCl}_{3}\right): \delta_{\mathrm{H}} 1.35(\mathrm{~d}, J 6.4 \mathrm{~Hz}, 3 \mathrm{H}, \mathrm{CH} 3), 2.25-2.37(\mathrm{~m}, 1 \mathrm{H}, \mathrm{CH}), 2.57-2.71(\mathrm{~m}, 1 \mathrm{H}, \mathrm{CH}), 2.73-3.83(\mathrm{~m}, 1 \mathrm{H}$, $\mathrm{CH}), 4.02-4.12(\mathrm{~m}, 1 \mathrm{H}, \mathrm{CH}), 4.83-4.95(\mathrm{~m}, 1 \mathrm{H}, \mathrm{CH}), 5.63-5.74(\mathrm{~m}, 1 \mathrm{H}, \mathrm{CH}), 6.01-6.13(\mathrm{~m}, 1 \mathrm{H}, \mathrm{CH}) .{ }^{13} \mathrm{C}$

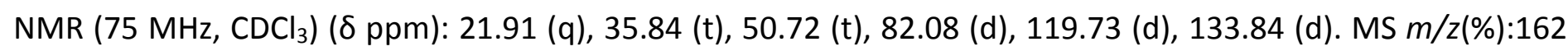
$\left[\mathrm{M}^{+}\right]$(2), $147(3), 98$ (8), 54 (100), 39 (50). Anal. Calcd for $\mathrm{C}_{6} \mathrm{H}_{10} \mathrm{O}_{3} \mathrm{~S}$ (162.20):C, 44.43; H, 6.21; S, 19.77. Found:C, 48.48; $\mathrm{H}, 6,24 ; \mathrm{S}, 19,66$.

Ethyl (1S,7S,8S)-4-oxa-3-thiabicyclo[5.1.0]octane-8-carboxylate 3,3-dioxide (6a). Compound 6a was prepared as colorless low-melting crystals according the general procedure from 6,7-dihydro-3H-1,2-oxathiepine 2,2dioxide (4a) (0.148 g, $1 \mathrm{mmol})$, ethyl diazoacetate (9) $(0.3 \mathrm{~mL}$ of ethyl diazoacetate in $5 \mathrm{~mL}$ DCM over $6 \mathrm{~h})$ and 2 mol\% of palladium diacetate (method A) $(0.004 \mathrm{~g})$ or Rhodium(II) acetate dimer $(0.0044 \mathrm{~g}, 1 \mathrm{~mol} \%)$ (Method B). Yield (A: $0.035 \mathrm{~g}, 15 \%, \mathrm{~B}: 0.077 \mathrm{~g}, 33 \%)$; colorless crystals; $\mathrm{mp}: 39-40{ }^{\circ} \mathrm{C} . \mathrm{IR}\left(\mathrm{KBr}, v_{\max }, \mathrm{cm}^{-1}\right): 2996,2981$, $1712,1397,1345,1151,963,891,860,729 .{ }^{1} \mathrm{H} N M R\left(\mathrm{CDCl}_{3}, 500 \mathrm{MHz}, \mathrm{COSY}, \mathrm{NOSY}\right): \delta_{\mathrm{H}} 1.25\left(\mathrm{t}, 3 \mathrm{H}, \mathrm{CH}_{3}\right), 1.52-$ $1.62(\mathrm{~m}, 2 \mathrm{H}, 2 \mathrm{CH}), 1.90(\mathrm{~m}, 1 \mathrm{H}, \mathrm{CH}), 2.10-2.33(\mathrm{~m}, 2 \mathrm{H}, 2 \mathrm{CH}), 2,33(\mathrm{~m}, 1 \mathrm{H}, \mathrm{CH}), 3.01(\mathrm{~m}, 1 \mathrm{H}, \mathrm{CH}), 3.81(\mathrm{~m}, 1 \mathrm{H}$, $\mathrm{CH}), 4.10\left(\mathrm{q}, 2 \mathrm{H}, \mathrm{CH}_{2}\right), 4.95(\mathrm{~m}, 1 \mathrm{H}, 1-\mathrm{H}) .{ }^{13} \mathrm{C} \mathrm{NMR}\left(\mathrm{CDCl}_{3}, 125 \mathrm{MHz}, \mathrm{HSQC}, \mathrm{HMBC}\right) \delta(\mathrm{ppm}): 14.09$ (q), 15.89 (d), 23.15 (d), $23.51(\mathrm{~d}), 48.18(\mathrm{t}), 60.92$ (t), 72.14 (t), 170.70 (s, C=O). MS m/z(\%): 234 [M+] (8), 206 (13), 189 (58), 155 (20), 126 (75), 79 (81), 67 (100). Anal. Calcd for $\mathrm{C}_{9} \mathrm{H}_{14} \mathrm{O}_{5} \mathrm{~S}$ (234.27): C, 46.14; H, 6.02; S, 13.69. Found: C, $46.21 ; H, 6.12 ; \mathrm{S}, 13.60$.

Ethyl (1S,5S,7S,8S)-5-methyl-4-oxa-3-thiabicyclo[5.1.0]octane-8-carboxylate 3,3-dioxide (6b). Compound 6b was prepared as colorless low melting colorless crystals according the general procedure from 7-Methyl-6,7- 
dihydro-3H-1,2-oxathiepine 2,2-dioxide (4b) (0.162 g, $1 \mathrm{mmol}$ ), ethyl diazoacetate (9) (0.3 $\mathrm{mL}$ of ethyl diazoacetate in $5 \mathrm{~mL}$ DCM over $6 \mathrm{~h}$ ) and 2 mol\% of palladium(II)acetate (method A) (0.004 g) or $1 \mathrm{~mol} \%$ of rhodium(II) acetate dimer (method B)(0.0044 g). Yield (A: 0.042 g, 17\%, B: 0.092 g, 37\%); colorless crystals; $\mathrm{mp}: 42-43{ }^{\circ} \mathrm{C}$. IR $\left(\mathrm{KBr}, v_{\max }, \mathrm{cm}^{-1}\right): 2982,1724,1367,1264,1164,1024,919 .{ }^{1} \mathrm{H} \mathrm{NMR}\left(\mathrm{CDCl}_{3}, 500 \mathrm{MHz}, \mathrm{COSY}\right.$, NOSY): $\delta_{\mathrm{H}} 1.28\left(\mathrm{t}, 3 \mathrm{H}, \mathrm{CH}_{3}\right), 1.40\left(\mathrm{~d}, 3 \mathrm{H}, \mathrm{CH}_{3}\right), 1.42-1.45(\mathrm{~m}, 2 \mathrm{H}, 2 \mathrm{CH}), 1.75-1.90(\mathrm{~m}, 2 \mathrm{H}, 2 \mathrm{CH}), 2,33(\mathrm{~m}, 1 \mathrm{H}, \mathrm{CH})$, $3.01(\mathrm{~m}, 1 \mathrm{H}, \mathrm{CH}), 3.81(\mathrm{~m}, 1 \mathrm{H}, \mathrm{CH}), 4.20\left(\mathrm{q}, 2 \mathrm{H}, \mathrm{CH}_{2}\right), 4.95(\mathrm{~m}, 1 \mathrm{H}, \mathrm{CH}) .{ }^{13} \mathrm{C} \mathrm{NMR}\left(\mathrm{CDCl}_{3}, 125 \mathrm{MHz}, \mathrm{HSQC}, \mathrm{HMBC}\right)$ $\delta(p p m): 14.16(q), 17.93$ (d), 21.51 (q), 23.70 (d), 31.25 (d), , 37.38 (t) $53.14(\mathrm{t}), 61.16(\mathrm{t}), 81.84(\mathrm{~d}), 171.08(\mathrm{~s}$, $\mathrm{C}=0$ ). MS m/z(\%): $248\left[\mathrm{M}^{+}\right]$(10), 207 (33), 93 (48), 67 (100), 41 (50). Anal. Calcd for $\mathrm{C}_{10} \mathrm{H}_{16} \mathrm{O}_{5} \mathrm{~S}$ (248.3): C, 48.37; $H, 6.50 ; S, 12.91$. Found: C, 48.41; H, 6.42; S, 12.89 .

Hex-5-en-2-yl ethenesulfonate (11a). Compound 11a was prepared as colorless oil according the general procedure from hex-5-en-2-ol (8a) (1.25 g, $12.5 \mathrm{mmol}), 2$-chloroethanesulfonyl chloride (9) (2.14 g, 1.05 equiv.) and $3 \mathrm{~mL}$ TEA. Yield (0.148 g, 79\%); Colorless oil; $\mathrm{R}_{f}$ : 0.73 (EtOAc: Isohexane, 1:4). IR ( $\left.\mathrm{KBr}, \mathrm{v}_{\max }, \mathrm{cm}^{-1}\right)$ : 3074, 2981, 2938, 1357, 1169, 894, 724. ${ }^{1} \mathrm{H} N M R\left(\mathrm{CDCl}_{3}, 300 \mathrm{MHz}\right): \delta_{\mathrm{H}} 1.48\left(\mathrm{~d}, J 6.3 \mathrm{~Hz}, 3 \mathrm{H}, \mathrm{CH}_{3}\right), 1.52-1.78(\mathrm{~m}$, $\left.2 \mathrm{H}, \mathrm{CH}_{2}\right), 2.12-2.21\left(\mathrm{~m}, 2 \mathrm{H}, \mathrm{CH}_{2}\right), 4.50-4.75(\mathrm{~m}, 1 \mathrm{H}, \mathrm{CH}), 4.48-5.15(\mathrm{~m}, 2 \mathrm{H}, 2 \mathrm{CH}), 5.60-5.80(\mathrm{~m}, 1 \mathrm{H}, \mathrm{CH}), 6.0(\mathrm{~d}, \mathrm{H}$,

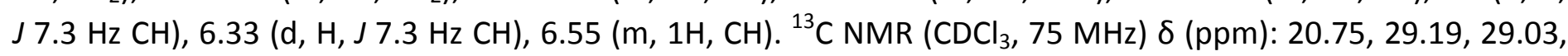

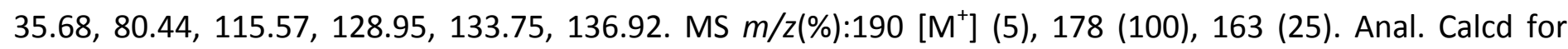
$\mathrm{C}_{8} \mathrm{H}_{14} \mathrm{O}_{3} \mathrm{~S}$ (190.26): C, 50.50; $\mathrm{H}, 7.42 ; \mathrm{S}, 16.85$. Found: $\mathrm{C}, \mathrm{C}, 50.42 ; \mathrm{H}, 7.50 ; \mathrm{S}, 16.76$.

Hept-6-en-3-yl ethenesulfonate (11b). Compound 11b was prepared according the general procedure from hept-6-en-3-ol (8b) (1.14 g, $10 \mathrm{mmol}), 2$-chloroethanesulfonyl chloride (9) (2.5 g, 1.5 equiv.) and $4 \mathrm{~mL}$ TEA Yield (0.137 g, 67\%); Pale yellow oil; $\mathrm{R}_{f}: 0.58\left(\mathrm{Et}_{2} \mathrm{O} /\right.$ Pentane: $\left.1 / 1\right) . \mathrm{IR}\left(\mathrm{KBr}, v_{\max }, \mathrm{cm}^{-1}\right): 2968,2938,1357,1162$, $985,755,723 .{ }^{1} \mathrm{H} N M R\left(\mathrm{CDCl}_{3}, 300 \mathrm{MHz}\right): \delta_{\mathrm{H}} 0.89\left(\mathrm{t}, 3 \mathrm{H}, \mathrm{CH}_{3}\right), 1.60-1.81\left(\mathrm{~m}, 4 \mathrm{H}, 2 \mathrm{CH}_{2}\right), 2.10-2.38\left(\mathrm{~m}, 2 \mathrm{H}, \mathrm{CH}_{2}\right)$, 4.50-4.61 (m, $1 \mathrm{H}, \mathrm{CH}), 5.0-5.22(\mathrm{~m}, 2 \mathrm{H}, 3 \mathrm{CH}), 5.6-5.75(\mathrm{~m}, 2 \mathrm{H}, 2 \mathrm{CH}), 6.10(\mathrm{~d}, J 7.8 \mathrm{HZ}, 1 \mathrm{H}, \mathrm{CH}), \quad 6.31(\mathrm{~d}, J 7.8 \mathrm{HZ}$, $\mathrm{H}, \mathrm{CH}), 6.515 .0(\mathrm{~m}, \mathrm{H}, \mathrm{CH}) .{ }^{13} \mathrm{C} \mathrm{NMR}\left(\mathrm{CDCl}_{3}, 75 \mathrm{MHz}, \delta \mathrm{ppm}\right): 9.11(\mathrm{q}), 27.22(\mathrm{t}), 29.03(\mathrm{t}), 34.06(\mathrm{t}), 85(\mathrm{~d})$, $115.34(\mathrm{t}), 128.71(\mathrm{t}), 133.78$ (d), 137.06 (d). $\mathrm{MS} \mathrm{m} / \mathrm{z}(\%): 189$ [M-CH$\left.{ }^{+}\right]$(2), 175 (79; 149 (100); 91(90) $\left[\mathrm{C}_{2} \mathrm{H}_{3} \mathrm{SO}_{2}^{+}\right] ; 55(95)\left[\mathrm{C}_{4} \mathrm{H}_{7}^{+}\right]$. Anal. Calcd for $\mathrm{C}_{9} \mathrm{H}_{16} \mathrm{O}_{3} \mathrm{~S}$ (204.28): C, 52.92; H, 7.89; S, 15.69. Found:C, 53.05; $\mathrm{H}, 7.95 ; \mathrm{S}, 15.72$

7-Methyl-6,7-dihydro-5H-1,2-oxathiepine 2,2-dioxide (12a). Compound 12a was prepared according the general procedure from hex-5-en-2-yl ethenesulfonate (11a) $(0.88 \mathrm{~g}, 5 \mathrm{mmol})$, Grubbs catalyst 2nd generation $(0.13 \mathrm{~g}, 0.03 \mathrm{eq})$ as a colorless crystals; Yield (75\%); Pale yellow oil. IR ( $\left.\mathrm{KBr}, v_{\max }, \mathrm{cm}^{-1}\right): 3064,1624,1332,1155$, 1131, 890, 813, 782, 637. ${ }^{1} \mathrm{H}$ NMR $\left(\mathrm{CDCl}_{3}, 600 \mathrm{MHz}\right): \delta_{\mathrm{H}} 1.37\left(\mathrm{~d}, J 6.2 \mathrm{~Hz}, 3 \mathrm{H}, \mathrm{CH}_{3}\right), 1.72-2.11\left(\mathrm{~m}, 2 \mathrm{H}, 2 \mathrm{CH}-\mathrm{CH}_{2}\right)$, 2.32-2.51 (m, 2H, 2CH-CH$), 4.48(\mathrm{~m}, 1 \mathrm{H}, \mathrm{CH}), 6.43-6.51\left(\mathrm{~m}, 2 \mathrm{H}, 2 \mathrm{CH}\right.$-vinyl). ${ }^{13} \mathrm{C} \mathrm{NMR}\left(\mathrm{CDCl}_{3}, 151 \mathrm{MHz}\right): 20.89$ (q), 25.12 (t), 29.95 (t), 80.70 (d), 129.44 (d), 140.57 (d). MS m/z(\%):162 [M+] (6), 119 (100), 79 (9.6), 54 (82), 39 (90). Anal. Calcd for $\mathrm{C}_{6} \mathrm{H}_{10} \mathrm{O}_{3} \mathrm{~S}$ (162.20): C, 44.43; H, 6.21; S, 19.77. Found: C, 44.48; H, 6,2; S, $19,66$.

7-Ethyl-6,7-dihydro-5H-1,2-oxathiepine 2,2-dioxide (12b). Compound 12b was prepared according the general procedure from hept-6-en-3-yl-ethenesulfonate (11b) $(0.60 \mathrm{~g}, 2.94 \mathrm{mmol})$ and Grubbs catalyst 2nd generation ( $0.078 \mathrm{~g}, 0.09 \mathrm{mmol}, 0.03$ equiv.) as a pale yellow oil. Yield (518 mg, 71\%); $\mathrm{R}_{f}: 0.31$ (Et ${ }_{2} \mathrm{O} / \mathrm{Pentane}$ 1/1); IR ( $\left.\mathrm{KBr}, v_{\text {max }}, \mathrm{cm}^{-1}\right): 2970,2935,1335,1156,891,638 .{ }^{1} \mathrm{H} \mathrm{NMR}\left(\mathrm{CDCl}_{3}, 300 \mathrm{MHz}\right): \delta_{\mathrm{H}} 0.91\left(\mathrm{t}, 3 \mathrm{H}, \mathrm{CH}_{3}\right)$, 1.50-1.62 (m, $1 \mathrm{H}, \mathrm{CH}), 1.75-1.92(\mathrm{~m}, 2 \mathrm{H}, 2 \mathrm{CH}), 2.10-2.20(\mathrm{~m}, 1 \mathrm{H}, \mathrm{CH}), 2.51-2.65(\mathrm{~m}, 2 \mathrm{H}, 2 \mathrm{CH}), 4.52-4.61(\mathrm{~m}, \mathrm{H}$, $\mathrm{CH}), 6.33-6.51(\mathrm{~m}, 2 \mathrm{H}, 2 \mathrm{CH}) .{ }^{13} \mathrm{C} \mathrm{NMR}\left(\mathrm{CDCl}_{3}, 75 \mathrm{MHz} ; \delta \mathrm{ppm}\right): 9.89(\mathrm{q}), 25.07(\mathrm{t}), 27.95(\mathrm{t}), 30.73(\mathrm{t}), 85.59(\mathrm{~d})$,

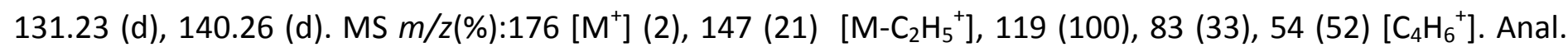
Calcd for $\mathrm{C}_{7} \mathrm{H}_{12} \mathrm{O}_{3} \mathrm{~S}$ (176.23): $\mathrm{C}, 47.71 ; \mathrm{H}, 6.86 ; \mathrm{S}, 18.19$. Found: $\mathrm{C}, 47.60 ; \mathrm{H}, 6.57 ; \mathrm{S}, 18.05$.

Ethyl (1S,4S,7S,8R)-4-ethyl-3-oxa-2-thiabicyclo[5.1.0]octane-8-carboxylate 2,2-dioxide(14). Compound 14 was prepared as colorless low-melting crystals with low yield (10\%) according the general procedure from 7ethyl-6,7-dihydro-5H-1,2-oxathiepine-2,2-dioxide (12b) (0.176 g, $1 \mathrm{mmol}$ ), ethyl diazoacetate (9) (0.3 $\mathrm{mL}$ of 
ethyl diazoacetate in $5 \mathrm{~mL}$ DCM over $6 \mathrm{~h}$ ) and (0.0044 g, 1 mol\%) rhodium(II) acetate dimer (method B). Yield (A: 0\%, B: .026 g, 10\%); colorless crystals; mp: $45-47^{\circ} \mathrm{C} . \mathrm{IR}\left(\mathrm{KBr}, v_{\max }, \mathrm{cm}^{-1}\right): 2972,1742,1334,1155,890 .{ }^{1} \mathrm{H}$ NMR $\left(\mathrm{CDCl}_{3}, 500 \mathrm{MHz}\right): \delta_{\mathrm{H}}$ 0.97-1.10 $\left(\mathrm{m}, 3 \mathrm{H}, \mathrm{CH}, \mathrm{CH}_{3}\right), 1.15-1.32\left(\mathrm{~m}, 4 \mathrm{H}, 2 \mathrm{CH}_{2}\right), 1.41\left(\mathrm{t}, 3 \mathrm{H}, \mathrm{CH}_{3}\right), 1.70-182(\mathrm{~m}$, $1 \mathrm{H}, \mathrm{CH}), 2.05-2.21(\mathrm{~m}, 1 \mathrm{H}, \mathrm{CH}), 2.51-2.62\left(\mathrm{~m}, 2 \mathrm{H}, \mathrm{CH}_{2}\right), 4.20-4.29\left(\mathrm{~m}, 2 \mathrm{H}, \mathrm{CH}_{2}\right), 4.22-4.32(\mathrm{~m}, 1 \mathrm{H}, \mathrm{CH}), 4.85-4.98$ $(\mathrm{m}, 1 \mathrm{H}, 1-\mathrm{H}) .{ }^{13} \mathrm{C} \mathrm{NMR}\left(\mathrm{CDCl}_{3}, 125 \mathrm{MHz} ; \delta \mathrm{ppm}\right): 11.22(\mathrm{q}), 14.17$ (q), $17.8(\mathrm{~d}), 20.90(\mathrm{~d}), 25.12(\mathrm{t}), 28.2(\mathrm{t}), 32.59$ (t), 61.05 (d), $62.1(\mathrm{t}), 68.20$ (d), 189.70 (s). MS m/z(\%):262 [M+] (2), 254 (33), 145 (48), 117 (100), 71 (50). Anal. Calcd for $\mathrm{C}_{11} \mathrm{H}_{18} \mathrm{O}_{5} \mathrm{~S}$ (262.32): $\mathrm{C}, 50.36 ; \mathrm{H}, 6.92 ; \mathrm{S}, 12.22$. Found: $\mathrm{C}, 50.16 ; \mathrm{H}, 6.78 ; \mathrm{S}, 12.31$.

2-Allylphenyl ethenesulfonate (16). Compound 16 was prepared according the general procedure from 2allylphenol (15) (1.34 g, $10 \mathrm{mmol}$ ), chloroethanesulfonyl chloride (9) (2.5 g, 1.5 equiv.) and $4 \mathrm{~mL}$ of TEA. Yield (1.75 g, 78\%); Pale yellow oil $\mathrm{R}_{f}$ : 0.55 (Et $\mathrm{E}_{2} \mathrm{O} /$ Pentane: 1/1); IR $\left(\mathrm{KBr}, v_{\max }, \mathrm{cm}^{-1}\right)$ : 3069, 1486, 1368, 1179, 1149, 870, 775, 756. ${ }^{1} \mathrm{H}$ NMR $\left(300 \mathrm{MHz}, \mathrm{CDCl}_{3}\right): \delta_{\mathrm{H}} 3.41-352\left(\mathrm{~m}, 2 \mathrm{H}, \mathrm{CH}_{2}\right), 5.12-5.23\left(\mathrm{~m}, 2 \mathrm{H},=\mathrm{CH}_{2}\right), 5.92-6.12(\mathrm{~m}, 1 \mathrm{H}$, $\mathrm{CH}), 6.22(\mathrm{~d}, 1 \mathrm{H}, \mathrm{CH}), 6.44(\mathrm{~d}, 1 \mathrm{H}, \mathrm{CH}), 6.75-6.90(\mathrm{~m}, 1 \mathrm{H}, \mathrm{CH}), 7.22-7.41(\mathrm{~m}, 4 \mathrm{H}, 4 \mathrm{CH}-\mathrm{Ar}) .{ }^{13} \mathrm{C} \mathrm{NMR}(75 \mathrm{MHz}$, $\mathrm{CDCl}_{3}$; $\left.\mathrm{ppm}\right): 34.11\left(\mathrm{CH}_{2}\right), 116.79\left(\mathrm{CH}_{2}\right), 121.99(\mathrm{CH}), 127.16(\mathrm{CH}), 127.23(\mathrm{CH}), 127.52(\mathrm{CH}), 130.89(\mathrm{CH})$, $131.23\left(\mathrm{CH}_{2}\right), 132.58(\mathrm{CH}), 133.02(\mathrm{CH}), 135.42(\mathrm{CH}), 147.53(\mathrm{CH}) . \mathrm{MS} m / z(\%): 224\left[\mathrm{M}^{+}\right](85), 133(91), 105$ (100), 77(51), 51 (20). Anal. Calcd for $\mathrm{C}_{11} \mathrm{H}_{12} \mathrm{O}_{3} \mathrm{~S}(224.27): \mathrm{C}, 58.91 ; \mathrm{H}, 5.39 ; \mathrm{S}, 14.30$. Found:C, 58.82; H, 5.46; S, 14.32.

5H-Benzo[f][1,2]oxathiepine 2,2-dioxide (17). Compound 17 was prepared as colorless crystals according to the general procedure from 2-allylphenyl ethenesulfonate (16) $(0.60 \mathrm{~g}, 2.67 \mathrm{mmol})$ and Grubbs catalyst 2nd generation $\left(0.078 \mathrm{~g}, 0.09 \mathrm{mmol}, 0.03\right.$ equiv. Yield $(0.47 \mathrm{~g}, 90 \%)$; colorless crystals Et ${ }_{2} \mathrm{O} /$ Pentane: $\left.1 / 1\right)$; . IR ( $\mathrm{KBr}$, $\left.v_{\max }, \mathrm{cm}^{-1}\right): 3054,1613,1351,1168,1147,878,767 .{ }^{1} \mathrm{H} N M R\left(\mathrm{CDCl}_{3}, 300 \mathrm{MHz}\right): \delta_{\mathrm{H}} 3.72-3.79\left(\mathrm{~m}, 2 \mathrm{H}, \mathrm{CH}_{2}\right), 6.35$ $(\mathrm{m}, 1 \mathrm{H}, \mathrm{CH}), 6.48-6.52(\mathrm{~m}, 1 \mathrm{H}, \mathrm{CH}), 7.21-7.45$ (m, 4H, 4CH-Ar). ${ }^{13} \mathrm{C} \mathrm{NMR}\left(\mathrm{CDCl}_{3}, \delta \mathrm{ppm}\right): 30.99,123.51,127.49$, 127.64, 129.46, 129.70, 132.03, 137.52, 148.23. MS m/z(\%):196 [M+] (26), 131 (100), 103 (26), 77 (22). Anal. Calcd for $\mathrm{C}_{9} \mathrm{H}_{8} \mathrm{O}_{3} \mathrm{~S}$ (196.22): C, 55.09; $\mathrm{H}, 4.11 ; \mathrm{S}, 16.34$. Found: $\mathrm{C}, 55.17 ; \mathrm{H}, 3.95 ; \mathrm{S}, 16.46$.

Crystal data of compound 17: $\mathrm{C}_{9} \mathrm{H}_{8} \mathrm{O}_{3} \mathrm{~S} M=196.22$. a $[\AA]=6.7227(5), \mathrm{b}[\AA ̊]=7.2890(5), \mathrm{c}[\AA]=9.6408(7), \alpha$ $\left[^{\circ}\right]=84.431(2), \beta\left[{ }^{\circ}\right]=85.660(2), \gamma\left[^{\circ}\right]=63.030(2), V[\AA ̊ 3]=418.78(5), T[K]=100(2)$. Figure 1 illustrates the structure as determent. Full data can be obtained on requested from CCDC (CCDC 1867516)

\section{Acknowledgements}

The authors acknowledge the Science and Technology Development Fund (STDF), Egypt for financial support of the research activities related to project; Project ID 25521.

\section{References}

1. Mondal, S. Chem. Rev. 2012, 112, 5339.

https://doi.org/10.1021/cr2003294

2. Ghandi, M.; Taghi Nazeri, M.; Kubicki, M. Tetrahedron 2013, 69, 4979.

https://doi.org/10.1016/j.tet.2013.04.018

3. Zhang, C.P.; Chen, Q.Y.; Guo, Y.; Xiao, J.C.; Gu, Y.C. Coord. Chem. Rev. 2014, 261, 28. https://doi.org/10.1016/j.ccr.2013.11.010

4. Bhowmik, B.B.; Ganguly, P. Spectrochim. Acta A Mol. Biomol. Spectrosc. 2005, 61, 1997. https://doi.org/10.1016/j.saa.2004.07.031 
5. Morimoto, Y.; Kurihara, H.; Kinoshita, T. Chem. Commun. 2000, 3, 189. https://doi.org/10.1039/a909094k

6. Natrajan, A.; Wen, D. Green Chem. Lett. Rev. 2013, 6, 237. https://doi.org/10.1080/17518253.2013.768708

7. Karsch, S.; Freitag, D.; Schwab, P. Synthesis 2004, 10, 1696.

8. Camarasa, M.J. Synthesis of 3'-Spiro-substituted Nucleosides: Chemistry of TSAO Nucleoside Derivatives. In Chemical Synthesis of Nucleoside Analogues; Merino, P., Ed.; John Wiley \& Sons: Hoboken, NJ, USA, 2013; Chapter 10, pp. 427-472. https://doi.org/10.1002/9781118498088.ch10

9. De Castro, S.; Familiar, O.; Andrei, G.; Snoeck, R.; Balzarini, J.; Camarasa, M.J.; Zquez, S.V. Chem. Med. Chem. 2011, 6, 686. https://doi.org/10.1002/cmdc.201000546

10. De Castro, S.; Peromingo, M.T.; Naesens, L.; Andrei, G.; Snoeck, R.; Balzarini, J.; Velazquez, S.; Camarasa, M.J .J. Med. Chem. 2008, 51, 5823.

https://doi.org/10.1021/im800050t

11. Hensel, A. Pharm. Acta Helv. 1995, 70, 25. https://doi.org/10.1016/0031-6865(94)00048-Z

12. Wang, P.L.; She, G.M.; Yang, Y.N.; Li, Q.; Zhang, H.G.; Liu, J.; Cao, Y.Q.; Xu, X.; Lei, H.M. Molecules 2012, 17, 4972.

https://doi.org/10.3390/molecules17054972

13. Wong, H. N. C.; Hon, M. Y.; Tse, C.W.; Yip, Y. C.; Tanko, J.; Hudlicky, T. Chem. Rev. 1989, 89, 165. https://doi.org/10.1021/cr00091a005

14. Doyle, M. P.; Forbes, D. C. Chem. Rev. 1998, 98, 911.

https://doi.org/10.1021/cr940066a

15. Lebel, H.; Marcoux, J. F.; Molinaro, C.; Charette, A. B. Chem. Rev. 2003, 103, 977. https://doi.org/10.1021/cr010007e

16. Pellissier, H. Tetrahedron 2008, 64, 7041. https://doi.org/10.1016/i.tet.2008.04.079

17. Doyle,M. P.; Winchester, W. R.; Hoorn, J. A.; Lynch, A. V.; Simonsen, S. H.; Ghosh, R. J. Am. Chem. Soc. 1993, 115, 9968.

https://doi.org/10.1021/ja00075a013

18. Nishiyama, H.; Itoh, Y.; Matsumoto, H.; Park, S. B.; Itoh, K. J. Am. Chem. Soc. 1994, 116, 2223. https://doi.org/10.1021/ja00084a104

19. Uchida, T.; Irie, R.; Katsuki, T. Synlett 1999, 1163. https://doi.org/10.1055/s-1999-2782

20. Che, C. M.; Huang, J. S.; Lee, F.W.; Li Y.; Lai T. S.; , Kwong, H. L.; Teng, P. F.; Lee, W. S.; Lo W. C.; Peng, S. M.; Zhou, Z. Y. J. Am. Chem. Soc. 2001, 123, 4119.

https://doi.org/10.1021/ja001416f

21. Huang, L. Y.; Chen, Y.; Gao, G. Y.; Zhang, X. P. J. Org. Chem. 2003, 68, 8179. https://doi.org/10.1021/jo0350880

22. Ali, K. A.; Jäger, A.; Metz, P. Arkivoc 2016, iii, 15. http://dx.doi.org/10.3998/ark.5550190.p009.359

23. Ali, K. A.; Mlostoń, G.; Urbaniak, K.; Linden A.; Heimgartner, H. J. Sulfur Chem. 2017, 37, 604. https://doi.org/10.1080/17415993.2017.1346103 
24. Abdel Hafez, N. A.; Ali, K. A.; Ibrahim A. A.; Elnaggar, D. H.; Sleem. A. A. Mini Rev. Med. Chem. 2018, 18, 1398.

https://doi.org/10.2174/1389557518666180530124509

25. Muravev, A.; Laishevtsev, Al.; Galieva, F.; Bazanova, O.; Ali, K. A.; Solovieva, S.; Antipin, I.; Konovalov, A. Macroheterocycles 2017, 10, 203.

https://doi.org/10.6060/mhc170304m

26. Ali, K. A.; Ragab E. A.; Dawood, K. M. Turk. J. Chem. 2016, 40, 277. https://doi.org/10.3906/kim-1503-75

27. Porer, Morris, Padgett, US. Patent WO 97/42185, 1997.

28. Dauban, P.; Dodd, R. Org. Lett. 2000, 2, 2327. https://doi.org/10.1021/ol000130c

29. Dawood, K. M.; Bramborg, A.; Darweesh, A.F.; Spinde, K.; Rogachev, V.; Jäger, A.; Metz, P. Heterocycles 2016, 93,723.

https://doi.org/10.3987/COM-15-S(T)64 